\title{
GRAPHS ORIENTABLE AS DISTRIBUTIVE LATTICES ${ }^{1}$
}

\author{
DWIGHT DUFFUS AND IVAN RIVAL
}

\begin{abstract}
There are two types of graphs commonly associated with finite (partially) ordered sets: the comparability graph and the covering graph. While the first type has been characterized, only partial descriptions of the second are known. We prove that the covering graphs of distributive lattices are precisely those graphs which are retracts of hypercubes.
\end{abstract}

Much of the combinatorial interest in finite ordered sets is linked to the properties of two types of undirected graphs commonly used to represent them: the comparability graph and the covering graph. For an ordered set $(P, \leqslant)$ the vertex set of both graphs is $P$. A pair $\{a, b\}$ of elements of $P$ is an edge of the comparability graph of $P$ if $a<b$; a pair $\{a, b\}$ is an edge of the covering graph $\mathcal{C}(P)$ of $P$ if $b$ covers $a$ [that is, $a<b$ and, if $a<c \leqslant b$, then $c=b$ ]. It is well known that $a$ graph $G$ is the comparability graph of an ordered set if and only if each odd cycle of $G$ has a triangular chord $[4,5]$. In contrast little is known about this question (cf. [9]): when is a graph the covering graph of an ordered set? The purpose of this note is to answer the question for a special class of ordered sets: finite distributive lattices.

Of course, every finite distributive lattice $D$ of length $n$ can, as an algebra, be embedded in the distributive lattice $2^{n}$ of all subsets of an $n$-element set; in fact, $\mathcal{C}(D)$ is a subgraph of (the hypercube) $\mathcal{C}\left(2^{n}\right)$. It is curious that the graph retracts of the hypercubes account for the covering graphs of all finite distributive lattices.

THEOREM. A finite graph $G$ is the covering graph of a distributive lattice of length $n$ if and only if $G$ is a retract of $\mathcal{C}\left(2^{n}\right)$ and $\operatorname{diam} G=n$.

Let $H=(V(H), E(H))$ and $G=(V(G), E(G))$ be graphs. An edge-preserving map $f$ of $H$ to $G$ is a map of $V(H)$ to $V(G)$ such that $\{f(a), f(b)\} \in E(G)$ if $\{a, b\} \in E(H)$. Call $H$ a retract of $G$ if there are edge-preserving maps $f$ of $H$ to $G$ and $g$ of $G$ to $H$ such that $g \circ f(a)=a$ for each $a \in V(H)$. (If $H$ is a retract of $G$ it is convenient to take $H$ as a subgraph of $G$ and $f$ to be an inclusion map.) Notice that every subgraph of $\mathcal{C}\left(2^{n}\right)$ is the covering graph of an ordered set. In fact, there are even nondistributive lattices whose covering graphs are subgraphs of $\mathcal{C}\left(\mathbf{2}^{n}\right)$. Both $H$ and $F$ are subgraphs of $G \cong \mathcal{C}\left(2^{3}\right) . H$ is a retract of $G$ and $H$ is the covering graph of a distributive lattice. $F$ is the covering graph of a nondistributive lattice and $F$ is not a retract of $G$.

Received by the editors March 5, 1981.

1980 Mathematics Subject Classification. Primary 06D99, 05 C75.

${ }^{1}$ This work was supported in part by N.S.E.R.C. Operating Grant No. A.4077. 


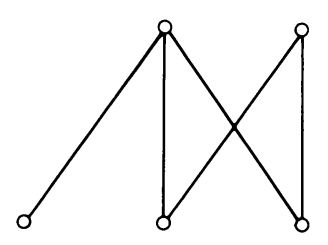

H

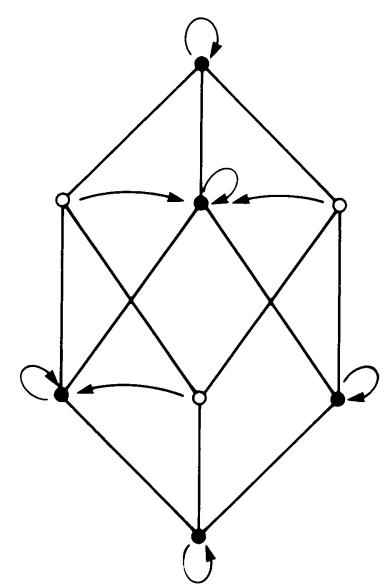

$G \cong C\left(2^{3}\right)$

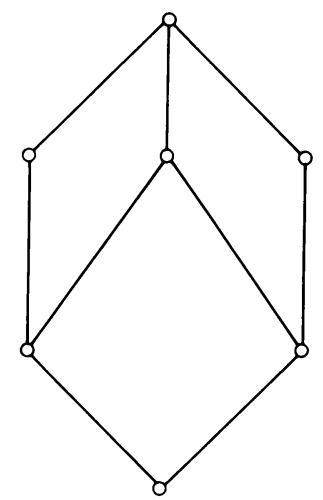

$F$

Proof of The Theorem. Several technical results are required for the proof.

Let $G$ be a finite connected graph and let $D$ be a finite distributive lattice. For $a, b \in V(G)$ we define the distance $d(a, b)$ of $a$ to $b$ as the number of edges in a path of minimum size in $G$ which contains $a$ and $b$. For a chain $C$ of $D$ we define its length by $l(C)=|C|-1$; the length $l(D)$ of $D$ is the maximum of the lengths of the chains of $D$. The height $h(a)$ of an element $a$ of $D$ is the length of the interval $\left[0_{D}, a\right]=\left\{x \in D \mid 0_{D} \leqslant x \leqslant a\right\}$, where $0_{D}$ is the least element of $D$.

Lemma 1 [3]. Let $D$ be a finite distributive lattice.

(i) If $a \leqslant b \leqslant c$ in $D$ then $d(a, c)=d(a, b)+d(b, c)$ in $C(D)$.

(ii) For all $a, b \in D, d(a, b) \geqslant|h(a)-h(b)|$ in $C(D)$.

(iii) For all $a, b \in D, d(a, b)=h(b)-h(a)$ in $C(D)$ if and only if $a \leqslant b$ in $D$.

For a finite connected graph $G$ we define the diameter diam $G$ of $G$ by

$$
\operatorname{diam} G=\sup \{d(a, b) \mid a, b \in V(G)\} .
$$

LEMMA 2 [2; cf. 3]. Let $D$ be a finite distributive lattice.

(i) $\operatorname{diam} C(D)=l(D)$ in $C(D)$.

(ii) If $d(a, b)=\operatorname{diam} \bigodot(D)$ in $\mathcal{C}(D)$ then there is a distributive lattice $D^{\prime}$ such that $\mathcal{C}\left(D^{\prime}\right)=\mathcal{C}(D)$, with least element $0_{D^{\prime}}=a$ and greatest element $1_{D^{\prime}}=b$.

LEMMA 3 [10]. Let $D$ be a maximal proper sublattice of a finite distributive lattice $D^{\prime}$ satisfying $l(D)=l\left(D^{\prime}\right)$. Then there is a cover-preserving sublattice $S$ of $D^{\prime}$ and elements $a \leqslant b$ in $S$ satisfying the following conditions:

(i) $D=D^{\prime}-[a, b]$;

(ii) $[a, b] \subseteq S$;

(iii) $y \in S$ if $y$ covers $x$ or $x$ covers $y$ for some $x \in[a, b]$;

(iv) there is an order isomorphism $\varphi$ of $S$ onto $\mathbf{2}^{2} \times[a, b]$ where $2^{2}=\{0<u, v<1\}$, such that $\varphi(x)=(u, x)$ for all $x \in[a, b]$. 
Let $G$ be a retract of $\mathcal{C}\left(2^{n}\right)$ and let $\operatorname{diam} G=n$; we may take $G$ to be a subgraph of $\mathcal{C}\left(\mathbf{2}^{n}\right)$. Let $g$ be an edge-preserving map of $\mathcal{C}\left(2^{n}\right)$ onto $G$ satisfying $g(a)=a$ for $a \in V(G)$. Let $d(a, b)=n$ in $G$. By 2(ii) there is a distributive lattice $D^{\prime}$ such that $\varrho\left(D^{\prime}\right)=\bigodot\left(2^{n}\right), 0_{D^{\prime}}=a$, and $1_{D^{\prime}}=b$. For $x, y \in V(G) \subseteq D^{\prime}$ let $x \vee y$ denote their supremum in $D^{\prime}$ and let $z=g(x \vee y)$. According to 1(i),

$$
d(a, x \vee y)+d(x \vee y, b)=d(a, b)=d(a, z)+d(z, b) .
$$

Since $g$ is edge-preserving, $d(a, z) \leqslant d(a, x \vee y)$ and $d(z, b) \leqslant d(x \vee y, b)$. Therefore, $d(a, z)=d(a, x \vee y)$ and $d(z, b)=d(x \vee y, b)$, so $h(z)=h(x \vee y)$ in $D^{\prime}$. Moreover, by 1(iii),

$$
d(x, z) \leqslant d(x, x \vee y)=h(x \vee y)-h(x),
$$

whence,

$$
d(x, z) \leqslant h(z)-h(x) .
$$

Again, by 1 (iii), $z \geqslant x$ in $D^{\prime}$; similarly, $z \geqslant y$ in $D^{\prime}$. As $h(z)=h(x \vee y)$ we conclude that $g(x \vee y)=z=x \vee y$. By duality, $g(x \wedge y)=x \wedge y$ so the elements of $G$ form a sublattice of $D^{\prime}$. Finally, by 2(i), this sublattice has length $n$.

Suppose now that $G$ is a graph and $G=\mathcal{e}(D)$ where $D$ is a distributive lattice of length $n$. By 2(i), diam $G=n$. The distributive lattice $D$ can be embedded as a sublattice in $2^{n}$; for convenience we may take $D$ to be a sublattice of $2^{n}$. Then there is a sequence $D=D_{0}, D_{1}, D_{2}, \ldots, D_{k}=2^{n}$ of distributive lattices such that $D_{i}$ is a maximal proper sublattice of $D_{i+1}$ and $l\left(D_{i}\right)=l\left(D_{i+1}\right)(i=0,1,2, \ldots, k-1)$. It is enough to show that if $D$ is a maximal proper sublattice of a finite distributive lattice $D^{\prime}$ and $l(D)=l\left(D^{\prime}\right)$ then $\mathcal{E}^{\prime}(D)$ is a retract of $\mathcal{C}^{\prime}\left(D^{\prime}\right)$.

Following the notation of 3(i), $D=D^{\prime}-[a, b]$ for some $a \leqslant b$ in $D^{\prime}$. Define a map of $g$ of $D^{\prime}$ to $D$ by $g(x)=x$ for all $x \in D$, and $g(x)=\varphi^{-1}((v, x))$ for all $x \in[a, b]$. To see that $g$ is edge-preserving let $x$ cover $y$ in $D^{\prime}$. If $x, y \in D$ then $g(x)=x$ covers $y=g(y)$ in $D$. If $x, y \in[a, b]$ then $g(x)=\varphi^{-1}((v, x))$ covers $\varphi^{-1}((v, y))=g(y)$. If $x \in[a, b]$ and $y \notin[a, b]$ then $y \in S$ and $\varphi(y)=(0, x)$ so $g(x)=\varphi^{-1}((v, x))$ covers $\varphi^{-1}((0, x))=\varphi^{-1}(\varphi(y))=y=g(y)$.

Remarks. 1. Actually, the proof of the Theorem shows this fact. Let $D$ be a finite distributive lattice and let $S \subseteq D$ with $\operatorname{diam} \varrho(S)=\operatorname{diam} \varrho(D)$. Then there is a distributive lattice $D^{\prime}$ such that $\mathcal{C}\left(D^{\prime}\right)=\mathcal{C}(D)$ and $S$ is a sublattice of $D^{\prime}$ if and only if $\mathcal{e}(S)$ is a retract of $\mathcal{C}(D)$.

2. Our theorem was suggested by this simple fact. A graph is the covering graph of an ordered set if and only if it is a retract of the covering graph of an ordered set.

3. In [2] L. Alvarez has shown that a finite graph $G$ is the covering graph of a finite distributive lattice if and only if $G$ satisfies the following conditions: (i) $G$ is connected and contains no odd cycles; (ii) there exist $a_{1}, a_{2} \in V(G)$ such that $\operatorname{diam} G=d\left(a_{1}, a_{2}\right)$ and if $\{c, e\},\{c, d\} \in E(G)$ with $d\left(a_{i}, e\right)=d\left(a_{i}, d\right)=d\left(a_{i}, c\right)+1$ then there is $a$ unique $f_{i} \in V(G)$ such that $d\left(a_{i}, f_{i}\right)=d\left(a_{i}, c\right)+2$ and $\left\{f_{i}, e\right\},\left\{f_{i}, d\right\} \in E(G)$, where $i=1$ or $i=2$; (iii) if $G$ contains a subgraph $S \cong F$ (see figure) then $S$ is contained in a subgraph $S^{\prime} \cong e\left(2^{3}\right)$; (iv) $G$ contains no subgraph isomorphic to the bipartite graph $K_{2,3}$. 
Other results concerning covering graphs of ordered sets are found in $[1,2,3,6,7$ and 8].

\section{REFERENCES}

1. M. Aigner and G. Prins, $k$-orientable graphs (preprint 1980).

2. L. Alvarez, Undirected graphs as graphs of modular lattices, Canad. J. Math. 17 (1965), 923-932.

3. D. Duffus and I. Rival, Path length in the covering graph of a lattice, Discrete Math. 19 (1977), $139-158$.

4. A. Ghouilà-Houri, Characterization of nonoriented graphs whose edges can be oriented in such a way as to obtain the graph of an order relation, C. R. Acad. Sci. Paris 254 (1962), 1370-1371.

5. P. C. Gilmore and A. J. Hoffman, A characterization of comparability graphs and of interval graphs, Canad. J. Math. 16 (1964), 539-548.

6. J. Jakubik, On lattices whose graphs are isomorphic, Czechoslovak Math. J. 4 (1954), 131-140.

7. Modular lattices of locally finite length, Acta Sci. Math. 37 (1975), 79-82.

8. K. M. Mosesjan, Basable and strongly basable graphs, Akad. Nauk Armjan. SSR Dokl. 55 (1972), $83-86$.

9. O. Ore, Theory of graphs, Amer. Math. Soc. Colloq. Publ., vol. 38, Providence, R.I., 1962; reprinted 1974.

10. I. Rival, Maximal sublattices of finite distributive lattices. II, Proc. Amer. Math. Soc. 44 (1974), 263-268.

Department of Mathematics, Emory University, Atlanta, Georgia 30322

Department of Mathematics, University of Calgary, Calgary, Alberta, T2N 1N4, Canada 